\title{
Hybrid Repair of Chronic Stanford Type B Aortic Dissection with Expanding Arch Aneurysm
}

\author{
Sheena Chen, MD, ${ }^{1,2}$ Kyongjune Benjamin Lee, MD,${ }^{1,2}$ Bao-Ngoc Nguyen, MD, ${ }^{1}$ Robyn Macsata, MD, ${ }^{1}$ \\ Shawn Sarin, MD, ${ }^{1}$ Gregory D. Trachiotis, $\mathrm{MD}^{1,2}$ \\ ${ }^{1}$ The George Washington University Hospital, Washington, DC; ${ }^{2}$ Washington D.C. Veterans Affairs Medical Center, Washington, DC
}

\section{ABSTRACT}

Purpose: The management of patients with chronic Stanford type B aortic dissection who develop complications requires intervention without clear guidelines. Chronic aortic dissection is difficult to treat and often leads to significant morbidity and mortality. We report a complex case of chronic Stanford type B aortic dissection (TBAD) with an expanding false lumen aneurysm and distal fenestrations that required a multi-stage hybrid repair.

Technique: The patient first underwent a median sternotomy for the ascending aorta to the innominate artery and innominate artery to the left carotid artery bypass, followed by a left carotid to left axillary artery bypass. Due to continued aneurysmal growth, the patient ultimately underwent total cervical and abdominal aortic debranching as well as thoracic and abdominal endovascular grafting with iliac excluders. The patient recovered well after the surgery and had no further expansion of the aneurysm at 12-month follow up.

Conclusion: Endovascular repairs have been the mainstay of chronic TBAD repair, but hybrid approaches may be necessary for difficult repairs. A multi-stage hybrid repair approach has been successful in a patient who had a chronic type $\mathrm{B}$ aortic dissection with aneurysmal degeneration that failed medical management.

\section{INTRODUCTION}

Chronic type B aortic dissection poses serious complication risks for patients, where complications arise from the progression of the unresolved dissection process causing aneurysmal expansion or progression of dissection [Fattori 2013]. Due to a lack of randomized controlled trials, the optimal management of Stanford type B aortic dissections (TBAD) remains contentious, in terms of the timing of repair and surgical techniques [Fattori 2013; Svensson 2008] While there is evidence to treat uncomplicated acute aortic dissection with medical therapy, the recommendations for uncomplicated TBAD have been controversial [Fattori

Received fuly 20, 2020; accepted August 25, 2020.

Correspondence: Gregory D. Trachiotis, MD, Cardiothoracic Surgery and Heart Center Veterans Affairs Medical Center, 50 Irving St. NW, Sec 112, Washington, DC 20422 (e-mail: gregory.trachiotis@va.gov).
2013; Svensson 2008]. Although optimal medical therapy is widely accepted as the standard of care for uncomplicated TBAD, many of these patients will develop complications, such as malperfusion, retrograde dissection, and aneurysmal degeneration that would require intervention [Fattori 2013; Svensson 2008; Hiratzka 2010; Hagan 2000]. It is estimated that the 5-year survival rate of patients with chronic type $\mathrm{B}$ aortic dissection on medical therapy is between $60 \%$ to $80 \%$ [Fattori 2013]. Treatment for patients with chronic TBAD who develop complications is particularly challenging regardless of approach, compared with patients who had surgical interventions at the acute phase of TBAD [Nauta 2016]. Here, we present a challenging multi-stage hybrid repair surgery for a patient with chronic TBAD that failed medical management and developed aneurysmal degeneration.

\section{CASE REPORT}

The patient was a 58-year-old male with hypertension, hyperlipidemia, obesity (body mass index of 42), obstructive sleep apnea, and a non-traumatic chronic Stanford type $\mathrm{B}$ aortic dissection that was first diagnosed four years prior to the initial surgery. The patient had a dissection of the descending thoracic with dissection flap just distal to the orifice of the left subclavian artery takeoff. The associated aneurysm at the origin left subclavian takeoff was initially stable on surveillance at $5 \mathrm{~cm}$. Despite optimal medical management, he developed aneurysmal dilation of the descending aorta to $6.5 \mathrm{~cm}$ with a dissection flap originating just distal

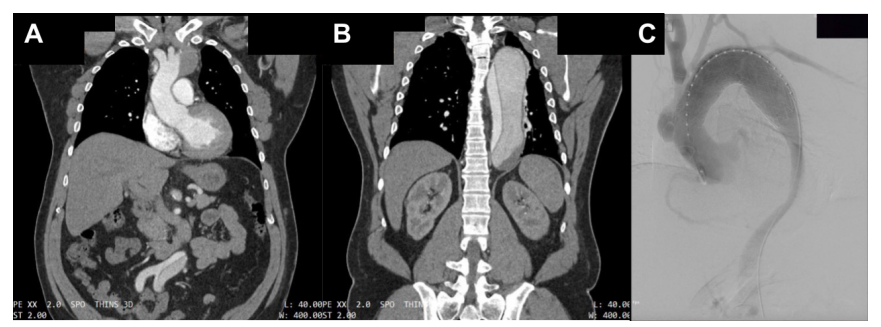

Figure 1. Coronal computed tomography (CT) image of the chronic type B aortic dissection with aneurysmal degeneration. A) The entry false lumen tear just distal to the level of left subclavian takeoff; B) The false lumen with associated aneurysm and an intramural thrombus; $C$ ) The aortic arch after debranching and TEVAR with good coverage of the entry tear. 


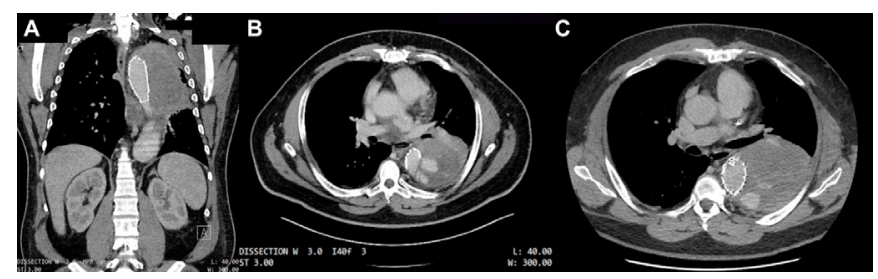

Figure 2. Post thoracic endovascular aortic repair (TEVAR) CT imaging. A) Coronal view of the $9 \mathrm{~cm}$ aneurysmal degeneration; B) The continued pressurization of aneurysm through the false lumen at the distal descending aorta; C) The same aneurysm after the extension of TEVAR graft and persistent flow of contrast into the aneurysm.

to the left subclavian artery takeoff and terminated in the left proximal common iliac artery. (Figure 1A) (Figure 1B) The operating team recommended an open repair for the patient; however, the patient refused due to his preference to avoid cardiopulmonary bypass and the lengthy recovery associated with an open repair.

After an extensive discussion amongst a multi-disciplinary team, including cardiothoracic surgery, vascular surgery, and interventional radiology, the decision was made to proceed with a hybrid repair, including a staged open cervical debranching (aorta to innominate artery bypass and left carotid to axillary artery bypass) and thoracic endovascular aortic repair (TEVAR) afterward for entry tear site coverage. The patient first underwent a median sternotomy for ascending aorta to innominate artery and innominate artery to left carotid artery bypass, followed by a left carotid to left axillary artery bypass, due to the friability of the left subclavian artery, with a $6 \mathrm{~mm}$ reinforced Polytetrafluoroethylene (PTFE) graft. A few days later, the patient had a spinal drain placement and underwent TEVAR with GORE@ TAG® thoracic stent graft $37-37 \mathrm{~mm}$ x $20 \mathrm{~cm}$ that covered the native origins of the innominate, left carotid, and left subclavian arteries (Figure 1C). The patient tolerated the two-stage procedure well and was discharged home.

The patient continued to be asymptomatic and the follow-up computer tomography (CT) scans remained stable. However, he developed new-onset back pain nine months after the initial repair, and a follow-up CT showed a growth in the descending thoracic aorta false lumen, now with a total aortic diameter expanded from $6.5 \mathrm{~cm}$ to $9 \mathrm{~cm}$ with continued pressurization of the aneurysm from a distal fenestration (Figures 2A and 2B). The patient was taken back to the operating room for an extension of TEVAR distally to the celiac artery with an overlying Zenith Alpha ${ }^{\mathrm{TM}}$ graft that was placed in the previous GORE® TAG ${ }^{\circledR}$ graft down to above the celiac artery. Angiogram after graft placement demonstrated that there was a second small fenestration by the iliac arteries. As the imaging showed that most of the descending false lumen was obliterated, the team decided to end the procedure and continue with medical management, understanding that there was a chance the patient would need a bifurcating graft at the iliac origin should the patient continue to have aneurysmal expansion or remain symptomatic.

Despite the intervention, the aneurysm had further enlargement of the false lumen aneurysm on CT scan (Figure

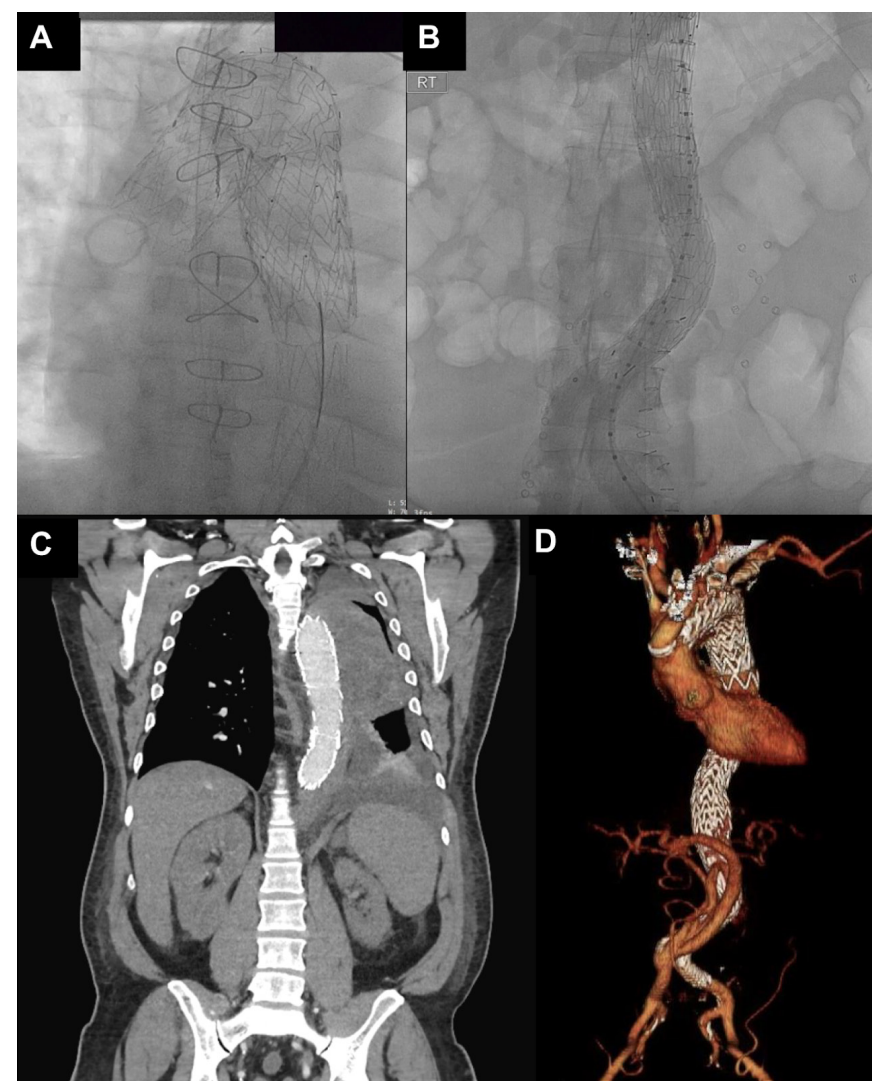

Figure 3. Post abdominal debranching and endovascular grafting of the abdominal aorta and placement of iliac excluder. A) The arch endograft placed in previous surgery with distal extension of endograft; B) No further retrograde perfusion of the false lumen through the distal fenestration at the iliac vessels was observed; C) Coronal view of six-month postoperative CT scan showing a stable false lumen aneurysm without further expansion or flow of contrast; D) A 3D reconstruction showing the endograft as well as the aorta to innominate debranching and abdominal debranching vessels originating from the right iliac artery.

2C). On the same admission, angiogram confirmed that the patient had continued retrograde flow into the false lumen at the level of the iliac arteries, and GORE® EXCLUDER ${ }^{\circledR}$ AAA Endoprosthesis graft was used to cover the exit tear at the iliac arteries. However, false lumen perfusion persisted from another proximal fenestration. The patient agreed to a hybrid operation. Subsequently, he underwent abdominal debranching with the right common iliac artery to the superior mesenteric artery, celiac artery, left and right renal arteries using a $14 \mathrm{~mm}$ main trunk Dacron graft with four separate limbs of $10 \mathrm{~mm}, 10 \mathrm{~mm}, 7 \mathrm{~mm}$, and $7 \mathrm{~mm}$, respectively. On postoperative day 2, a thoracic stent graft was placed via femoral access in the visceral aorta, spanning from the previously inserted abdominal aortic stent-grafts to the previously inserted thoracic stent graft. Completion angiogram showed exclusion of the false lumen and exclusion of the proximal and distal fenestrations (Figures 3A and 3B). The patient's postoperative CT angiogram at one year showed contrast opacification in the false lumen, which remained stable (Figures $3 \mathrm{C}$ and $3 \mathrm{D}$ ). 


\section{DISCUSSION}

The general consensus on uncomplicated type B aortic dissection is to manage patients conservatively with optimal medical therapy [Fattori 2013; Nauta 2016]. However, several studies have shown that up to $40 \%$ of patients eventually will need intervention and $20 \%$ of patients will develop false lumen aneurysms despite aggressive medical therapy [Diethrich 2008]. Complicated chronic TBAD with aneurysmal degeneration presents additional surgical challenges when undergoing open repair, due to extensive aortic replacement, need for circulatory arrest and aortic clamping, and increased risk of spinal cord injury, contributing to a significant in-hospital mortality rate of near 30\% [Hagan 2000; Diethrich 2008].

Endovascular repairs have been the mainstay of chronic TBAD repair, but hybrid approaches may be necessary for difficult repairs. The goal of endovascular therapy is to cover the entry dissection tear and cause thrombosis of the false lumen [Hagan 2000; Diethrich 2008; Roselli 2015]. However, in chronic TBAD, sealing of the entry tear often is inadequate to ensure false lumen thrombosis due to distal fenestrations [Hagan 2000]. Distal fenestrations allow persistent pressurization of the false lumen, and the desired remodeling of the vasculature with endovascular repair does not occur to satisfactory levels [Hagan 2000; Nauta 2016]. Chronic TBAD results in increased rates of endoleak, need for reintervention, extensive endograft extension and debranching, and retrograde dissections [Roselli 2015].

In our reported case, the chronic nature of the disease with certain anatomic features suggested that the patient may not do well with standard endovascular therapy and likely would have needed further intervention after the initial TEVAR. The extension of the dissection down to the bilateral iliac vessels increased the likelihood of distal fenestrations, contributing to the continued pressurization of the false lumen after TEVAR. Similar findings were observed in studies where false lumen thrombosis and remodeling in chronic dissection was successful after TEVAR when the dissection was limited to the thoracic aorta as opposed to when the dissection extended more distally [Roselli 2015; Kang 2011]. In respect to the visceral vessels, our patient's left renal artery and the celiac artery both were partially supplied by the false lumen, making the persistent pressurization of the false lumen more likely.

At the time of discussion of surgical options, these findings were presented to the patient to explain that he likely was to have a more durable outcome with open surgical repair compared with endovascular repair. Due to the patient's strong preference to avoid extensive open surgery involving circulatory arrest, we elected to proceed with the hybrid repair. In retrospect, the patient ended up requiring three hospitalizations with five separate procedures, including one open sternotomy, one open abdominal surgery, and three endovascular procedures. An argument could be made that the patient might have been better served with an initial open thoracic aortic aneurysm repair with cardiopulmonary bypass, however, there is no certainty that patient would not have needed additional surgeries afterward. Considering the patient's strong preference and viable surgical option via a hybrid approach, the operating team provided the patient with a safe and suitable option.

In terms of the merit for intervention for an uncomplicated chronic TBAD case that our patient presented with, there have been studies that have identified patients who present with an aortic diameter greater than $40 \mathrm{~mm}$ and with patent false lumen have a significantly higher risk of developing complications [Fattori 2013; Svensson 2008; Nauta 2016]. However, most guidelines recommend surgical intervention only for patients who develop complications with chronic TBAD, including recurrence of symptoms, aneurysmal dilation $>55 \mathrm{~mm}$, or yearly increase of $>4 \mathrm{~mm}$ in aortic diameter [Fattori 2013]. An argument could be made to preemptively repair uncomplicated chronic TBAD for patients who are considered high risk for future development of complications.

Based on the current data, surgical intervention is often times necessary in addition to endovascular repairs for chronic TBAD, as the long-term data showed that patients who underwent earlier and more aggressive interventions fared better in long-term follow up [Fattori 2013; Svensson 2008; Hagan 2000; Nauta 2016; Roselli 2015]. As we understand more about the disease process of TBAD, it is imperative to identify patients with high-risk presentations of TBAD who may benefit from early intervention. The goal of early identification and intervention also should be included in clinical guideline considerations to prevent chronic degeneration of aortic dissection in this patient population when the disease process is more manageable.

\section{CONCLUSION}

Endovascular repairs have been the mainstay of chronic TBAD repair, but hybrid approaches may be necessary for difficult repairs. A multi-stage hybrid repair approach is a viable option in patients who had chronic type B aortic dissections with aneurysmal degeneration that failed medical management.

\section{REFERENCES}

Diethrich EB, Ramaiah VG, Kpodonu J, et al. 2008. Case 16: Endovascular Management of Acute Stanford Type B Dissection. Endovascular and Hybrid Management of the Thoracic Aorta: A Case-based Approach. Blackwell Publishing Ltd., Hoboken, NJ. 104-113.

Fattori R, Cao P, De Rango P, et al. 2013. Interdisciplinary expert consensus document on management of type B aortic dissection. Journal of the American College of Cardiology. Apr 23;61(16):1661-1678.

Hagan PG, Nienaber CA, Isselbacher EM, et al. 2000. The International Registry of Acute Aortic Dissection (IRAD): new insights into an old disease. JAMA. 283(7): 897-903.

Hiratzka LF, Bakris GL, Beckman JA, et al. 2010. 2010 ACCF/AHA/ AATS/ACR/ASA/SCA/SCAI/SIR/STS/SVM Guidelines for the diagnosis and management of patients with thoracic aortic disease. A Report of the American College of Cardiology Foundation/American Heart Association Task Force on Practice Guidelines, A. Journal of the American College of Cardiology. 55(14):e27-129. 
Kang WC, Greenberg RK, Mastracci TM, et al. 2011. Endovascular repair of complicated chronic distal aortic dissections: intermediate outcomes and complications. Journal of thoracic and cardiovascular surgery. 142(5):1074-83.

Nauta FJ, Trimarchi S, Kamman AV, et al. 2016. Update in the management of type B aortic dissection. Vascular Medicine. 21(3): 251-63.
Roselli EE. 2015. Thoracic endovascular aortic repair versus open surgery for type-B chronic dissection. Journal of Thoracic and Cardiovascular Surgery. 149(2):S163-7.

Svensson LG, Kouchoukos NT, Miller DC, et al. 2008. Expert consensus document on the treatment of descending thoracic aortic disease using endovascular stent-grafts. The Annals of thoracic surgery. 85(1):S1-41. 